\title{
The force magnitude of a human bite measured at the molar intercuspidation using fiber Bragg gratings
}

\author{
Ilda Abe, Maura S. Milczewski, Mauren A. Souza \\ Federal University of Technology - Paraná, 80230-901 Curitiba, Brazil, e-mail: ildaabe@utfpr.edu.br \\ Hypolito J. Kalinowski \\ Fluminense Federal University, 24210-240 Niterói, Brazil \\ Oscar F. Machuca, Gabriela C. Marin, Elisa S. Camargo \\ Pontifical Catholic University of Paraná, 80215-901 Curitiba, Brazil
}

\begin{abstract}
This study describes a localized method - using optical fiber Bragg gratings (FBGs) - to determine the human bite force at the very moment of occlusion, in maximum intercuspidation. The FBG was encapsulated in a silicone splint between uppers and lowers first molars and avoiding pre-contact with the other teeth in a dentition model. Gypsum dental castings of two volunteers are performed in a semi-adjustable articulator in the occluded situation to maintain angulations of the occlusal plane within normal occlusion. The sensor was characterized in vitro for both volunteers and the human bite force is measured in vivo. The orthodontic forces on the sensor are acquired at four moments: before of occlusion (free situation), occlusion, biting and maximum bite. The measured maximum bite forces are $85 \mathrm{~N}$ for female (brachyfacial) volunteer and $76 \mathrm{~N}$ for male (mesofacial) volunteer, between right uppers and lowers first molars. The difference can be associated to the facial pattern. The results obtained show the possibility to employ the optical encapsulated sensor to perform measurements of orthodontic forces in order to analysis different types of facial patterns with minimum of interference at occlusion.
\end{abstract}

Index Terms - fiber Bragg gratings, human bite force, in vivo measurements, optical fiber sensors.

\section{INTRODUCTION}

The need to measure the human bite process raises many interesting questions in fields like anatomy, physiology, nutrition, dentistry and pharmacology. Therefore, the interest in studying the biting process relies in knowing the relationships between the force magnitude and the correct occlusion, anatomy and teeth position. Additionally, it provides knowledge to optimize dental prostheses or implants, and to explain dental traumas or bruxism. It can also help to clarify the comprehension of oral diseases and mastication dysfunctions [1]-[3].

Occlusion is the relationship involving teeth contacts in a complex system of integrated structures, maxillary joint and neuromuscular system, which are responsible for the function of mastication, related to the opening and closing of the mouth [1],[2]. The masticatory function is studied in terms of mandible opening and closing (occlusion), temporomandibular joint activity, dental anatomy, 
dental position and muscle contraction [3].

The magnitude of bite force has been studied in animals and in humans employing different methods of measurements. The first experimental study defining intraoral forces is dated by 1681 , when it was reported the use of a specific gnathodynamometer for this purpose, as cited in [2]; the dimensions associated with this type of instrument are greater than $10 \mathrm{~mm}$ in the intraoral part, which interferes with the occlusion. In the $20^{\text {th }}$ century the bite force was studied with several types of mechanical devices; and since the 1950s, it has been studied employing electronic devices [2]. Methods like electromyography, which measure muscles activity with electrodes have been widely used until nowadays. More recently, studies were performed by combining muscle sedation with appropriate drugs, in order to understand their effects on the bite force and how this may influence the feeding process. Moreover, there is expressive research aimed to food industry, considering materials and texture, in order to develop new foods suitable to human and animal feeding [4]. Another recent study used magnetic field to measure bite force in humans [5].

The strain-gages devices are based in electrical resistance and the literature also reports the use of such tools to measure maximum human bite forces. A great range of forces has been disclosed, from 50 to $800 \mathrm{~N}$ [2]. The great variability of results for maximum bite force can be associated to several factors such as: age, gender, body mass index, temporomandibular disorders, strength of the muscles, craniofacial morphology, occlusal state, periodontal condition and psychological factors [2]. The study performed by Fernandes et al. [6] published the use of a strain-gauge transducer to determine maximum bite force in male and female volunteers, and their results reported values of $100 \mathrm{~N}$ and $75 \mathrm{~N}$, respectively. More recently in 2009, Bonakdarchian et al. had shown results of maximum bite forces of adults with normal occlusion and the effect of facial shapes on it [7]. Marquezin et al. shows the use of strain gages to measure bite forces in primary children dentition in comparison to preschool children [8].

The great force magnitudes associated to the methods proposed in the literature can generate interference points in several teeth, since it is sensitized by the proprioception, i.e., the people sensitivity in perceiving that there is something unknown in his body. It shall be recalled that electrical strain-gages need to be connected in a 4-wire scheme, which introduces an extra volume in the oral cavity. Multiple-point sensing (several teeth positions, e.g.) would become uncomfortable for the patient and the masticatory function would be compromised. The mentioned perception can alter the results of real bite forces in one tooth. In this case, a point sensor, with small dimensions is preferable.

Optical fiber Bragg gratings (FBGs) have wide potential for biomechanical and biomedical applications [9]. They have several advantages when compared to conventional resistive, piezoelectric or other solid-state sensing technologies. The FBGs have small dimensions and weight - the typical FBG has a length of $3 \mathrm{~mm}$ and a fiber diameter of $250 \mu \mathrm{m}(125 \mu \mathrm{m}$ without coating). FBG can be multiplexed, allowing spatially quasi-distributed measurements. Some characteristics make FBGs 
particularly attractive for in vivo purposes. Optical fiber is a non-electric and non- conductive device, and it has immunity to electromagnetic noise and radio frequency interference. FBGs have higher temperature and pressure capability compatible with human physiology figures. FBGs are flexible and they can adapt to complex contours, apart from being less intrusive as well. The fibers are made of silicate glass, which is biocompatible; furthermore, FBGs can be embedded into polymer or composite materials to be used in instruments requiring sterilization. Optical fiber sensors also present small risk for infection. FBGs are resistant to corrosive environments, and FBGs are also resistant to water and chemicals related to in vivo measurements.

Real time, in vivo, biomechanical measurements with FBG sensors were demonstrated at the beginning of the century [10],[11], attracting attention in several associated problems from ex vivo to in vivo conditions. In dentistry, there are many reports in the literature that employ Bragg gratings to monitor orthodontic forces [11]-[15]. Tjin et al. reported at first time the use of FBG sensors in odontology; the authors demonstrated the monitoring pressure and temperature of the dental splint as a function of time for apnoea patients [11]. Milczewski et al. evaluated the forces at several teeth as a function of the load applied; the results showed that it is possible to use either FBGs written in high birefringence fiber or a polymeric fiber microbend sensor, placed between the bracket and the surface of the tooth for verification of the orthodontic appliances [12],[13]. In another work, Milczewski et al. showed the use of the FBGs sensors in an artificial maxilla model, and they reported the possibility of monitoring the forces on the internal parts of the teeth and at the position where the strain takes place within the maxilla [14]. Franco et al. demonstrated the potential of the FBG in the measurements of different jaw movements, performed for patients with occlusal parafunction by using occlusal splints [15]. Marin et al. reported previous analysis of the use of FBG sensors to measure the magnitude of a human bite at the molar intercuspidation [16].

The estimated bite force largely depends on the number of occlusal contacts used during the specific measurement methods. The comprehension of the masticatory function relies on the understanding of different diseases, pains, malocclusion, dysfunctions and knowledge to the treatment like bone and dental prostheses or implants. The need for a punctual load sensor with low influence at the occlusion is essential in all these studies and assessments.

In this study, we present the use of punctual Bragg gratings sensors encapsulated in a silicon material placed at both first molar teeth for its characterization in vitro, based on a set up with gypsum models. Additionally, the in vivo characterization is also performed, in order to be used in human dentition studies. The importance of this system relies in measuring only the bite force transmitted from one tooth to another at the moment of occlusion and biting.

\section{MATERIAL AND METHODS}

A female brachyfacial volunteer, 25 years old and a male mesofacial volunteer, 32 years old, were selected, both with normal occlusion and without previous orthodontic treatment. 
Gypsum dental castings of both volunteers were performed in a semi-adjustable articulator, type Arcon, in the occluded situation. For a correct positioning of the maxilla (upper jaw) in relation to the mandible (lower jaw) average values were used, the horizontal condylar inclination at $30^{\circ}$ and the Bennett side shift at $15^{\circ}$.

The sensors are based on Bragg gratings inscribed in standard telecommunications fiber with $125 \mu \mathrm{m}$ of diameter. FBGs were written using an Excimer KrF laser (Coherent, Xantos XS 500) at $248 \mathrm{~nm}$, whose beam illuminates the fiber through a phase mask. Phase masks with spatial pitch of $1073.5 \mathrm{~nm}$ and $1067.4 \mathrm{~nm}$ were used in the inscription. The fibers samples were hydrogen loaded $\left(100 \mathrm{~atm}, 25^{\circ} \mathrm{C}, 4\right.$ days) before the grating production. The laser was operated at $5 \mathrm{~mJ}$ per pulse with $200 \mathrm{~Hz}$ repetition rate. The estimated length of the gratings is approximately $3 \mathrm{~mm}$, derived from the laser beam diameter and from an iris placed before the phase mask.

The acquisitions of optical spectra were performed in the writing process of the gratings, and along the in vitro and in vivo experiences using the amplified spontaneous emission of an Erbium doped fiber as a broadband light source, an optical circulator and an optical spectrum analyzer (Yokogawa, AQ6375) set to $50 \mathrm{pm}$ of resolution.

The sensor was involved in hot melted silicone and, using the gypsum casting, it was precisely positioned at the contact point during the occlusal surface of the lower first molar. The occlusal markings are obtained by using articulating paper, leaving on the cast a dot mark based on the contact point (Fig.1 (a)). The fiber was positioned on the silicon, near to the oral cuspids, and then a new layer of the silicon was added. Promptly the upper casting was occluded maintaining the intercuspidation between lower and upper molars, while the silicone was still hot (Fig.1 (b) and (c)). One sensor for each side of mandible (Fig.2 (a)) was produced, which were positioned on the first molars, right and left side (Fig.2 (b)).

\section{In vitro characterization}

The in vitro study is performed with loads from 0 to $90 \mathrm{~N}$ applied over the occluding model in defined sides (Fig.3 (a)). The mechanical system consists of a lever and scale masses of $0.50 \mathrm{~kg}$; the loads are applied with the help of a metal sphere, in specific positions at right and left side of the gypsum model (as shown in Fig.3 (b) and (c)). Fig.3 (b) depicts a diagram of the first right molar and the forces lines that are acting at the structure; the vertical dotted line represents the load direction, aligned with the human body in standing conditions; the leaning dotted line marks the alignment of the occlusal plane edge at the gypsum model. The loads are applied in two positions, right and left on the cast, when the sensor is inserted at each side of the gypsum model. The same procedure is done with the female and male occluding models.

Even if the loads in the mechanical system are applied along with the vertical human body direction, there are components of the applied force also along two perpendicular directions on the occlusal plane. The fiber sensor is located at the first molars contact point, i.e. in parallel to the occlusal plane. For this reason, the detected forces by the sensors are due to a longitudinal strain 
component; and the peak wavelength of the reflection spectrum moves to longer wavelengths (red shift). Higher load forces applied to the system can also induce birefringence at the fiber, due to the transversal components of the force. Therefore, the longitudinal forces induce longitudinal strains at the FBG sensors and it is possible to analyze the variation of the force loads by the shift of the spectral reflection band of the sensor.

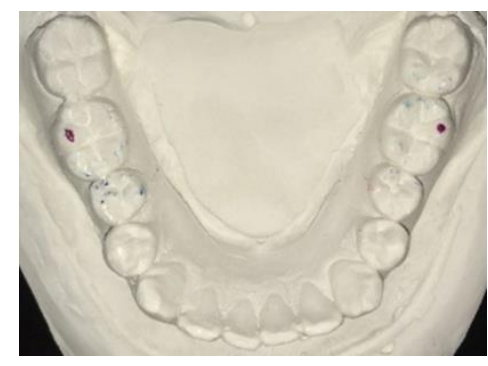

(a)

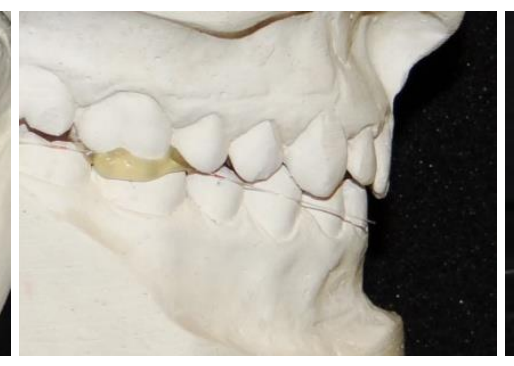

(b)

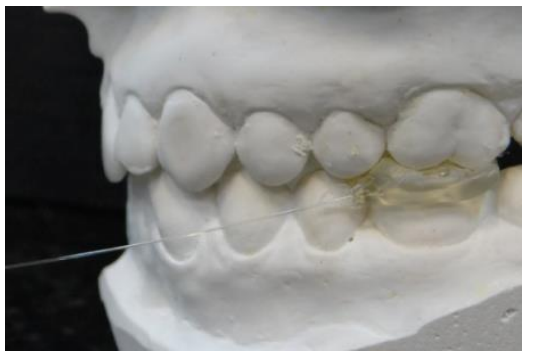

(c)

Fig. 1. (a) Gypsum cast model and contact point of both first molars; (b) sensor placed in position at right side; (c) at left side.

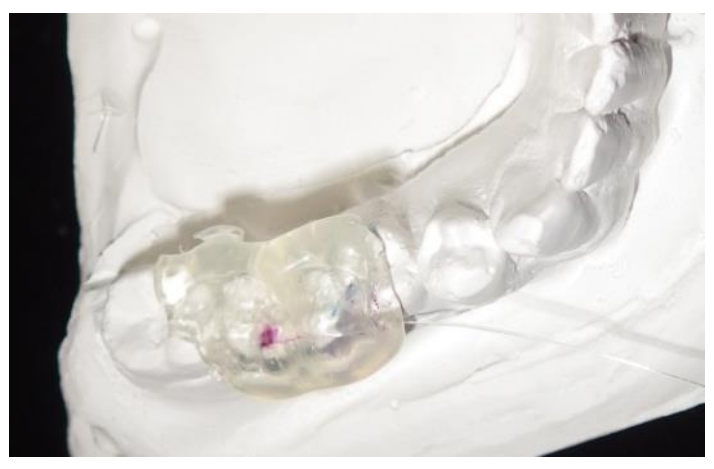

(a)

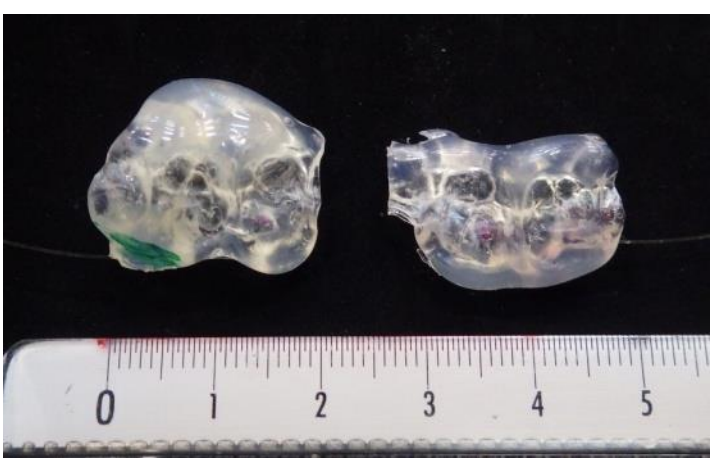

(b)

Fig. 2. (a) Sensor for bite force measurements formed by a FBG involved in silicone; (b) details of the silicone mold for the right and left sensors.

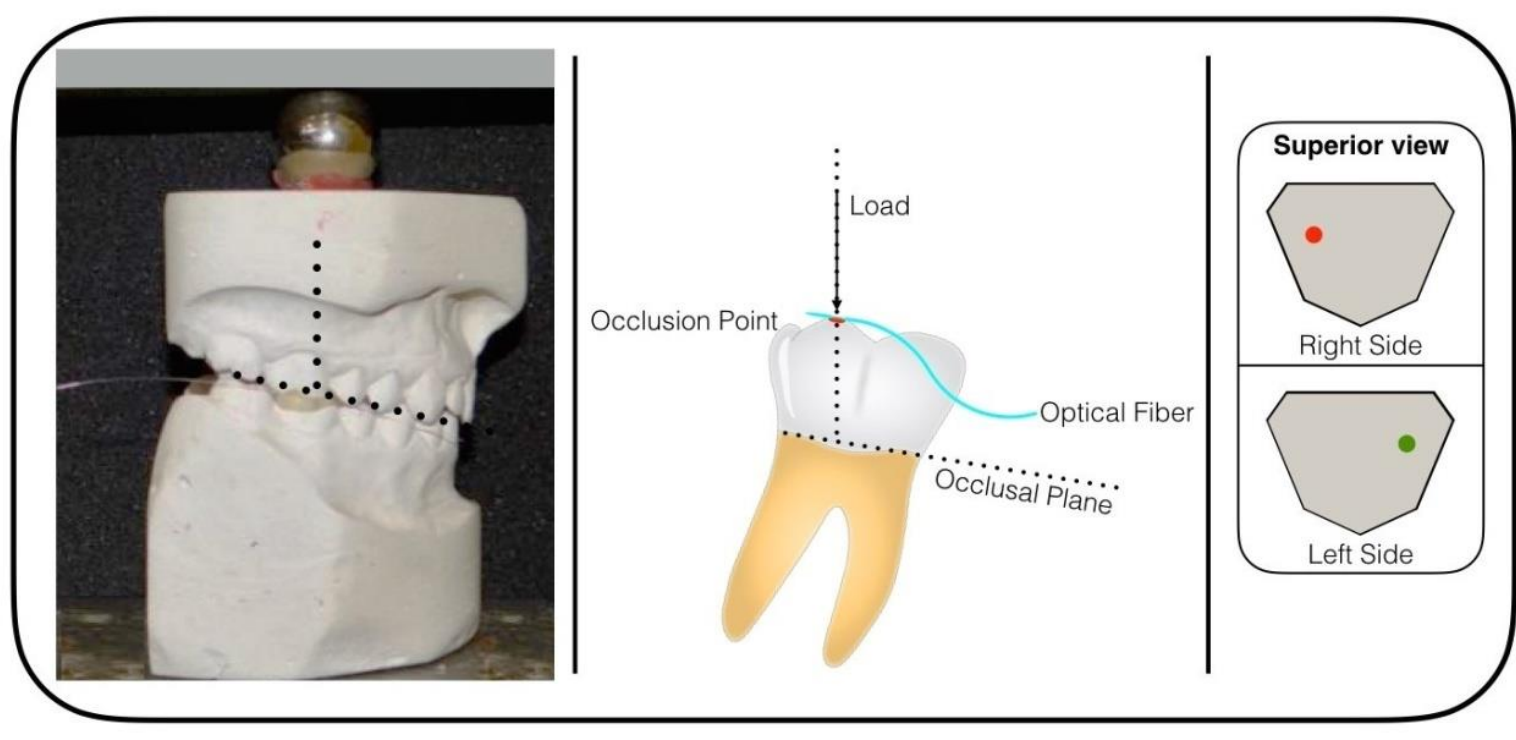

(a)

(b)

(c)

Fig. 3. (a) Gypsum model occluding with the sensor and loads being applied. (b) Diagram representing the first right molar, the optical sensor and the occlusal plane. (c) Superior view of the casts and the precision points for their load application. 


\section{In vivo characterization}

After the production of the encapsulated sensor and in vitro characterization, the sensor was inserted inside the mouth of both volunteers for the in vivo evaluation. The encapsulated sensor is positioned at the actual same occlusion point as performed within the cast; then the same process is applied to both volunteers (Fig.4 (a) and (b)).

The spectra of the gratings were obtained only for the right side of the mouth in vivo analysis, because the in vitro characterization shows that the preferred bite side in both volunteers is the right side. Measurements are performed with the sensor in four bite stages, which are: with the mouth open (before occlusion), with the teeth in occlusion, slightly biting and maximum biting. In between the biting stages, the volunteer was instructed to return to the stage before occlusion, i.e. keeping the mouth open. The spectrum of the grating is monitored under such condition until the reflection spectrum returned to its initial position, in order to assure that no hysteresis would affect the subsequent measurements.

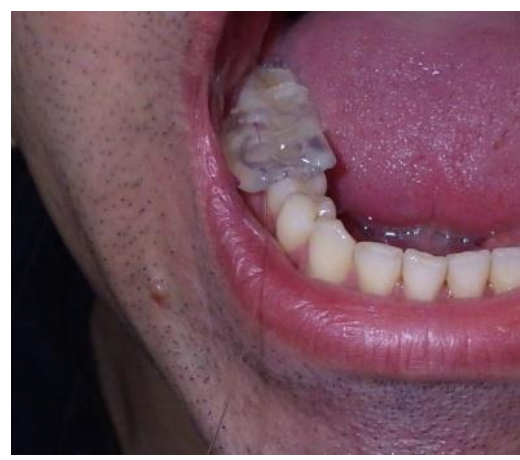

(a)

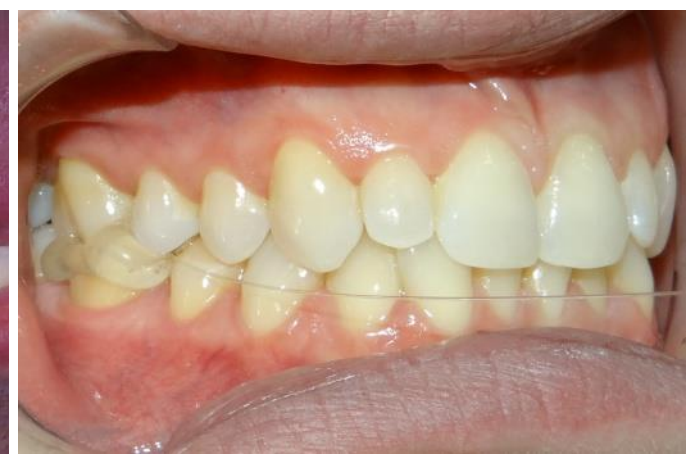

(b)

Fig. 4. (a) Encapsulated sensor positioned inside the mouth (male volunteer). (b) Encapsulated sensor between the first molars (female volunteer) at the right side of the mouth.

When the sensor is inserted in the mouth, there is an initial shift in the spectral position of the grating resonance band due to the increase of temperature, from room temperature to internal body temperature. A waiting interval of approximately $2 \mathrm{~min}$ is used, until the thermo-stability of the sensor is achieved. All the tests are performed in a very short time, about 5 min to complete all stages of measurements. During this period, no significant temperature changes are observed, despite of the initial thermo-stability.

\section{RESULTS \\ In vitro}

Initial tests of bite forces are performed in a static occlusion model, in order to obtain the sensitivities of the sensor. Several spectra of FBGs are obtained for different loads, which are applied over the model in the two positions, right and left on the castings, for both right and left sides of the sensors location. This characterization is performed for both male and female models. 
Fig.5 (a) and (b) shows the results for the female model. The curves describe the shift of the FBG spectral position as a function of the applied load, for right and left sensors, respectively. The sensitivity values are obtained from the linear best fitting to the curve. From the slope of the obtained best fit line it is possible to determine the force as a function of the applied load.

When the sensor is positioned in the right side, values of about $0.011 \mathrm{~nm} / \mathrm{N}$ were obtained, applying loads on the right side; and $0.001 \mathrm{~nm} / \mathrm{N}$ applying loads on the left side. On the other hand, when the sensor is located between left molars, the values were $0.009 \mathrm{~nm} / \mathrm{N}$ to loads applied on the left side and $0.002 \mathrm{~nm} / \mathrm{N}$ when the loads were applied on the right side. The results indicated that the side with best intercuspidation and dominant mastication for this female volunteer is the right side; as a consequence, the left side for the current volunteer, is the balancing side for the mastication process.

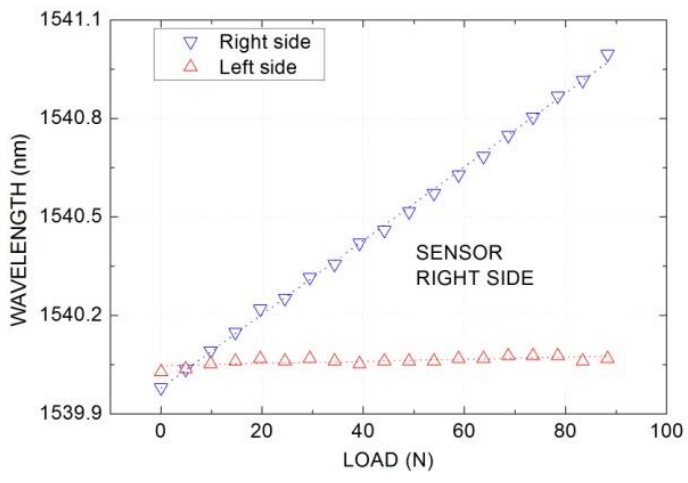

(a)

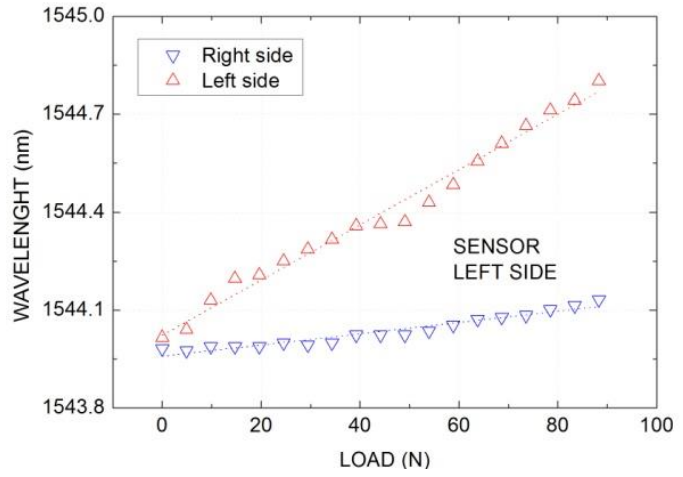

(b)

Fig. 5. Spectral position of the FBG resonance band as function of loads applied for the female model, at right and left side, with the sensors located: (a) in the right; and (b) in the left first molars. (The symbols represent the experimental data; and the dotted lines are their linear best fit).

Fig.6 (a) and (b) resumes the graphs with maximum response for both volunteers: sensor located at upper and lower right first molar and load applied at right side; sensor localized at upper and lower left first molar and load applied at left side. The displayed results in the graphs on Fig.6 show that the rights are the dominant sides for both volunteers.

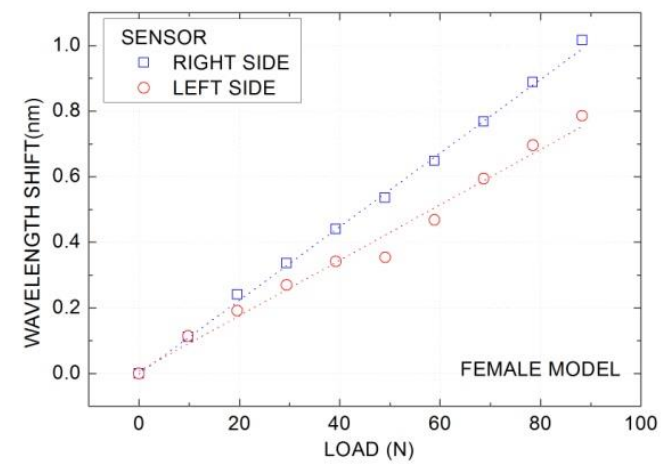

(a)

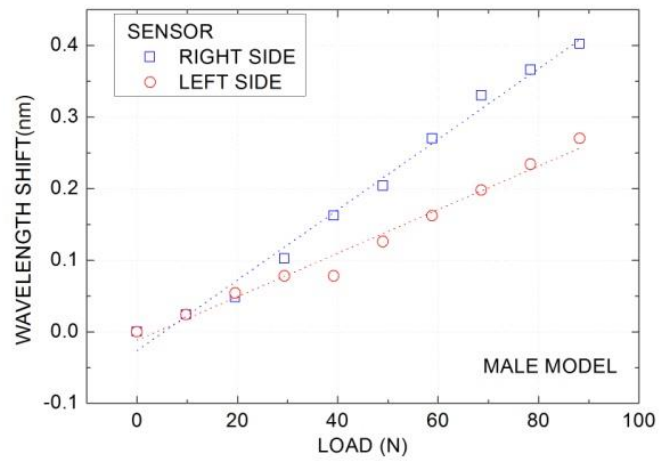

(b)

Fig. 6. Spectral shift of the sensor resonance as a function of the applied load on the right and left sides for: (a) the female model and (b) the male model. (The symbols are the experimental data; and the dotted lines are the linear best fit). 
Table 1 shows the sensitivity coefficient determined for the female and the male volunteers, showing the two sides (right and left) of the gypsum casts. The maximum values of bite force can be related to the occlusal plane, since the values are different for the female and male volunteer. The female brachyfacial volunteer shows a higher sensitivity values than the male mesofacial volunteer. This fact could be related to the facial pattern of volunteers; the brachyfacial volunteer has a smaller inclination of occlusal plane than the mesofacial pattern volunteer, and the orthogonal components of the forces for the brachyfacial pattern are greater than the mesofacial pattern, where the forces are distributed in orthogonal and longitudinal components.

TABLE I. LOAD SENSITIVITY FOR BOTH VOLUNTEERS, ACCORDING TO THE POSITION OF THE APPLIED LOADS AT THE RIGHT AND THE LEFT SIDES OF THE CAST MODELS, WHEN THE ENCAPSULATED SENSOR IS LOCALIZED AT UPPER AND LOWER RIGHT AND LEFT FIRST MOLAR.

\begin{tabular}{ccc}
\hline Sensor & Load & Sensitivity $(\mathrm{nm} / \mathrm{N})$ \\
\hline Right side (female volunteer) & Right side & 0.011 \\
Left side (female volunteer) & Left side & 0.009 \\
Right side (male volunteer) & Right side & 0.005 \\
Left side (male volunteer) & Left side & 0.003 \\
\hline
\end{tabular}

In vivo

From the wavelength shift of the FBG spectral position, it is possible to determine, using the previous measured sensor sensitivity, the forces applied by each volunteer during the occlusion and biting. Fig. 7 shows the obtained FBG reflection spectra for both volunteers, female volunteer (Fig.7 (a) and male volunteer (Fig.7 (b)) when the encapsulated sensor is inside of the mouth and located at upper and down first right molar. The position of each FBG reflection band can be associated to a different force applied by the volunteer, using the previously determined sensitivity.

It is possible to notice at the reflection spectrum of the grating that there are distortions in the band at biting positions - the effect is more evident at the short wavelength side of spectra from the female volunteer (Fig.7 (a)). The distortion is due to the birefringence induced by the masticatory force on the sensor, which partially splits the spectral band. The effect is, obviously, more important at maximum bite, since the transversal strain component is bigger. In the overall, the observed results prove that the Bragg grating encapsulated sensor can be used to measure the maximum bite forces. At the maximum bite, it is noticed that the transversal components (band splitting) are not superior to the longitudinal components (band shift). 


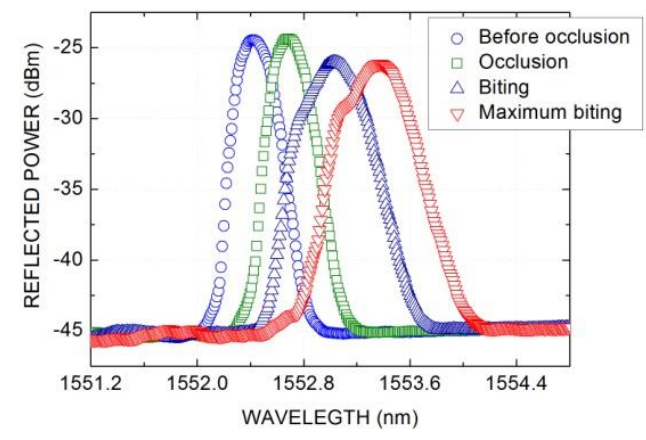

(a)

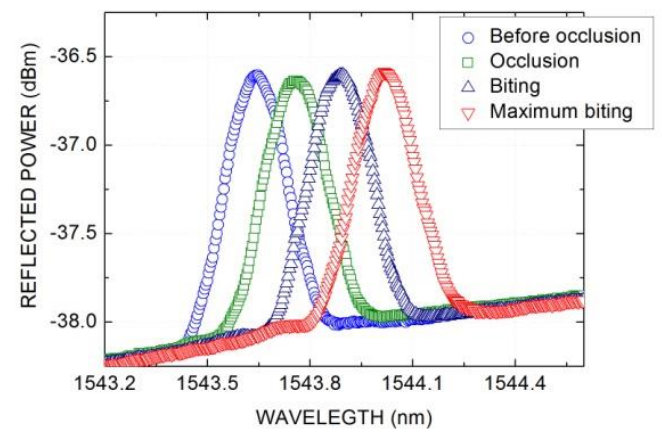

(b)

Fig. 7. In vivo evaluation: FBG optical reflection spectra for sensor at first right molar of (a) female and (b) male volunteers, at different stages (before occlusion, occlusion and biting).

Table 2 shows the values of force related to the four stages for both volunteers. The values are obtained from the reflection spectral band shift using the sensitivity coefficient for the right side of the mouth. The stage "before occlusion" is the initial point of the evaluation and the force value is considered to be zero at this point.

TABLE II. VALUES OF BITE FORCES FOR BOTH (FEMALE AND MALE) VOLUNTEERS.

\begin{tabular}{ccc}
\hline Stage & Female volunteer & Male volunteer \\
& Force $(\mathrm{N})$ & Force $(\mathrm{N})$ \\
\hline Before occlusion & 0 & 0 \\
Occlusion & 24 & 24 \\
Biting & 55 & 76 \\
Maximum biting & 85 & 76 \\
\hline
\end{tabular}

Based on the wavelength shift of the FBG spectrum, and using the previous measured sensor sensitivity, it is possible to determine the forces applied by the volunteers during the maximum bite. The bite force values obtained in vivo evaluations show that the female brachyfacial volunteer has a higher bite force than the male mesofacial volunteer. These values can be related to the face pattern of the volunteers and the distribution of the forces related to each type of occlusion. This is in according with the results obtained in vitro evaluations that shows different sensibility coefficients for each face pattern.

In comparison with earlier studies, the forces here obtained present a very large range, but this can be related to uncontrolled variables like age, gender and problems associated to the occlusion. In despite this, Hattori et al. [17] reported forces values of approximately $100 \mathrm{~N}$, using another method of measure. That value agrees with our data for punctual forces measurements. In addition, Fernandes et al., [6] also showed forces of $100 \mathrm{~N}$ and $75 \mathrm{~N}$, for male and female volunteers, respectively, 
obtained with strain gauge sensors. These values also confirm the magnitude values obtained in the present research.

\section{CONCLUSION}

In this paper we presented the evaluation of a new punctual sensor based in encapsulated FBG for measuring the bite forces aiming in vivo analyses. The advantage of using a FBG sensor is the possibility of obtaining the force values distributed in a restricted area at the contact point of occlusion and not an average value over a wide area, which is usually adopted by other methods reported in the literature. The FBG sensor characteristics show the feasibility of its use for allowing analyses of occlusal diagnostic in real case studies.

The characterization in vitro shows that the preferred bite side in both volunteers is the right side. Therefore the in vivo tests were carried out deliberately on this side. The sensibility coefficients values are higher for the female volunteer in comparison with the male volunteer.

The maximum values of bite force can be related to the occlusal plane, since the values are different for the female and male volunteer. On the other hand, the occlusal plane can be related to the facial pattern, because the brachyfacial pattern, related to the female volunteer, shows a higher force than the male volunteer, which has a mesofacial pattern. The brachyfacial pattern volunteer has a minor inclination of occlusal plane, in relation to the line force, than the mesofacial pattern volunteer.

The comprehension of the masticatory function and the level of the bite force measured in a real case study can confirm previous results using different methods and sensors, and might help to elucidate details within the masticatory process to be further applied in other dental studies.

\section{ACKNOWLEDGMENT}

The authors acknowledge CAPES, CNPq, FUNDAÇÃO ARAUCARIA, FINEP, UTFPR and PUC-PR for scholarships and funding. Prof. Kalinowski thanks the Federal University of Technology - Paraná, for receiving him as a voluntary researcher during part of time used in this work.

\section{REFERENCES}

[1] T. M. Graber and J. R. Vanarsdall, "Orthodontics principles and practice", W B Saunders Company, Philadelphia and London, 1963.

[2] D. Koc, A. Dogan and B. Bek, "Bite force and influential factors on bite measurement: A literature review", European Journal of Dentistry, vol. 4, pp. 223-232, 2010.

[3] S. Tomita, N. Matsuura and T. Ichinohe, "The combined effects midazolam and propofol sedation on muscle power" Anaesthesia, vol. 68, pp. 478-483, 2013.

[4] J. F. Meulenet and R. K. Gandhapuneni, "Development of the Bite Master II and is application to the study of cheese hardeness", Physiology \& behavior, vol. 89, pp. 39-43, 2006.

[5] A. D. Lantada, C. G. Bris, P. L. Morgado and J. S. Maudes, "Novel System for Bite-Force Sensing and Monitoring Based on Magnetic Near Field”, Communication Sensors, vol. 12, pp. 11544-11558, 2012.

[6] C. P. Fernandes, P. J. Glantz, S. A. Svensson and A. Bergmark, "A novel sensor for bite force determinations". Dental Materials, vol. 19: pp. 118- 126, 2003

[7] M. Bonakdarchian, N. Askari and M. Askari. "Effect of face form on maxilar molar bite force with natural dentition". Archives of Oral Biology, vol. 54: pp. 201-204, 2009.

[8] M. C. S. Marquezin, F. Y. Kobayashi, A. B. M. Montes, M. B. D. Gavião and P. M. Castelo, "Assessment of masticatory performance bite force, orthodontic treatment need and orofacial dysfunction in children and adolescents", Archives of oral biology, vol. 58, pp. 286-292, 2013.

[9] H. J. Kalinowski, I. Abe, J. A. Santos and A. Ramos, "Application of fibre Bragg grating sensors in biomechanics", Trends in Photonics, Transworld Research Network, Trivandrum, India, 2010. 
[10] G. Werhle, P. Nohama, H. J. Kalinowski, P. I. Torres and L.C. G. Valente, A fibre optic Bragg grating strain sensor for monitoring ventilatory movements". Measurement and Science Technology, vol. 12, pp. 805-809, 2001.

[11] S. C. Tjin, Y. K. Tan, M. Yow and J. Hao, "Recording compliance of dental splint use in obstructive sleep apnea patients by force and temperature modeling". Medical and Biology Engineering and Computing, vol. 39, pp. $182-184$. 2001.

[12] M. S. Milczewski, J. C. C. Silva, I. Abe, A. S. Paterno, J. A. Simões and H. J. Kalinowski, "Measuring Orthodontic Forces with HiBi FBG Sensors". Proceedings of 18th Optical Fiber Sensors Conference, Cancun, pp. 23-27, 2006.

[13] M. S. Milczewski, M. Stevenson, J. Canning, C. Mattelli and H. J. Kalinowski, "Sensitivity of silica and polymer microstructured fibres transversal pressure". Glass Technology, vol. 50, pp. 211-213, 2009.

[14] M. S. Milczewski, J. C. C. da Silva, C. Martelli, L. Grabarski, I. Abe and H. J. Kalinowski,. "Force Monitoring in a Maxilla Model and Dentition Using Optical Fiber Bragg Grating"s. Sensors, vol. 12, pp. 11957-11965, 2012.

[15] A. P. G. O. Franco, A. O. Gebert, M. A. Souza, L. Jeranoski, H. J. Kalinowski and I. Abe, "Measurement of mandibular movements in parafunctional patient using occlusal splint with Bragg gratings: pilot study", Proceedings SPIE, vol. 9916, Sixth European Workshop on Optical Fibre Sensors, 991618, 2016.

[16] G. C. Marin, M. S. Milczewski, I. Abe, S. C. P. S. Lopes, E. S. Camargo and H. J. Kalinowski, "The magnitude of a human bite measured exactly at the molar intercuspidation using FBG". Proceedings SPIE, vol. 9157, 24th International Conference on Optical Fibre Sensors, Santander, pp. 91575J-1-91575J-4, 2014.

[17] Y. Hattori, C. Satoh, S. Seki, Y. Watanabe, Y. Ogino and M. Watanabe, "Occlusal and TMJ loads in subjects with experimentally shortened dental arches”, Journal of Dental Research, vol. 82, pp. 532-536, 2003. 